\title{
Cavitary Covid Lesions - An Unusual Imaging Feature
}

\author{
Rajasbala Pradeep Dhandeㄹ, Suresh Vasant Phatak², Gaurav Ved Prakash Mishra ${ }^{3}$, Vaishali Patil Dhawan ${ }^{4}$, Soumya Jain ${ }^{5}$
}

1, 2, 3, 4, 5 Department of Radiodiagnosis, JNMC, Sawangi (Meghe) Wardha, Maharashtra, India.

\section{INTRODUCTION}

On January 30, 2020, the coronavirus disease 2019 (Covid-19), formerly known as the 2019 novel coronavirus (2019-nCoV), was declared as a global health emergency by the World Health Organization.

Coronavirus disease 2019 (Covid-19) is an infectious disease caused by severe acute respiratory syndrome coronavirus 2 (SARS-CoV-2). Imaging plays an essential role in the evaluation of Covid-19, with chest computed tomography (CT) being the major modality in diagnosing and managing Covid-19 pneumonia.

Common signs of Covid-19 pneumonia on chest CT scan are ground-glass opacities, consolidation, nodules, and linear opacities, halo and reverse halo signs. It can be accompanied by a "crazy-paving" pattern, air bronchograms, pleural hypertrophy, and pleural effusion. Cavitations are known to occur but are rare presentations. There are cases reported in the literature of the development of pulmonary cavity after bacterial infection in the late recovery stage in SARS patients. We present a case report of this rare entity in Covid-19 infection in a previously stable patient.

\section{PRESENTATION OF CASE}

A 50-year-old man with a diagnosis of Covid-19 presented to the hospital with fever and multiple episodes of intermittent cough and shortness of breath for five days. There was no history of haematemesis, epistaxis, chills, night sweats or weight loss. CT scan of the chest revealed bilateral multifocal patchy airspace disease with surrounding ground-glass opacities (GGO) consistent with Covid-19 pneumonia. Along with that, extensive areas of central cavitation with mild dilatation and thickening of bronchioles were noted.

Physical examination revealed few coarse crackles bilaterally. Infectious diseases, rheumatology and pulmonology consultants recommended an extensive workup including mycobacterial, autoimmune, HIV and fungal tests that were rendered negative. Peak C-reactive protein level was raised and peak oxygen requirement was noted to be $6 \mathrm{~L} / \mathrm{min}$. Reverse transcription-PCR assays for severe acute respiratory syndrome- coronavirus-2 (SARS-CoV-2) was persistently positive. Bronchoscopy was performed, and bronchoalveolar lavage samples had no growth.
Corresponding Author:

Dr. Soumya Jain,

Department of Radiodiagnosis, AVBRH, JNMC, DMIMS, Sawangi (Meghe) Wardha - 442001, Maharashtra, India.

E-mail: soumya2403@gmail.com

DOI: $10.14260 /$ jemds/2021/647

How to Cite This Article:

Dhande RP, Phatak SV, Mishra GVP, et al. Cavitary covid lesions- an unusual imaging feature. J Evolution Med Dent Sci 2021;10(36):3188-3191, DOI: 10.14260/jemds/2021/647

Submission 27-05-2021,

Peer Review 23-07-2021,

Acceptance 03-08-2021,

Published 06-09-2021.

Copyright (C) 2021 Rajasbala Pradeep Dhande et al. This is an open access article distributed under Creative Commons Attribution License [Attribution 4.0 International (CC BY 4.0)] 


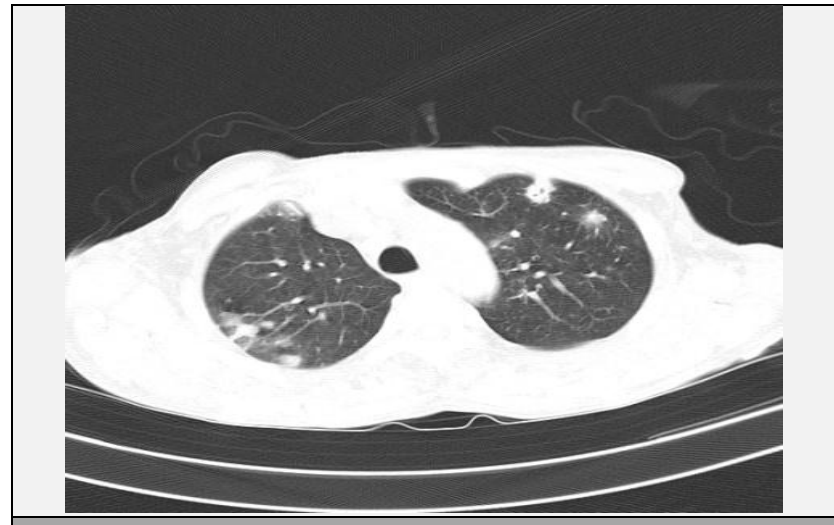

Figure 1. Axial Section Lung Window of the Upper Lung Fields Showing Patchy Ground Glass Opacities with Few Consolidations Containing Air Bronchogram
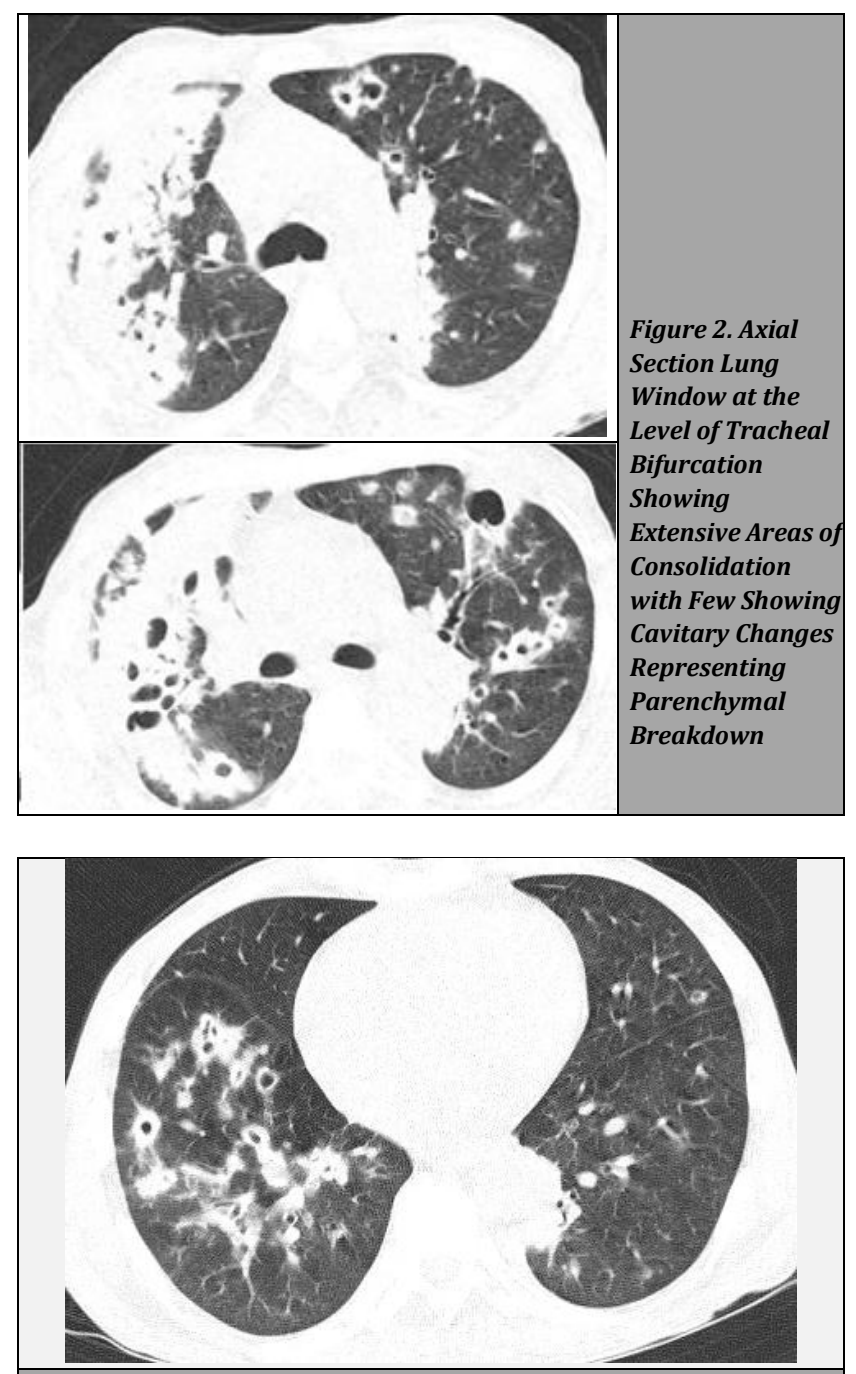

Figure 3. Axial Section Lung More Caudally Showing Cavitary Changes in the Right Lung Field with Surrounding Consolidation

\section{DISCUSSION}

Covid-19 transmits across individuals through respiratory droplets during close contact. A wide spectrum of clinical manifestations is seen with Covid-19, with the most common being fever ( $80.4 \%$ ), cough (63.1\%), fatigue (46\%), and expectoration (41.8\%). Other symptoms include anorexia
(38.8\%), chest tightness (35.7\%), shortness of breath (35 $\%$ ), dyspnoea (33.9\%), and muscle soreness (33\%). Olfactory dysfunction (41.0\%) and gustatory dysfunction (38.2 \%) are also frequently present. Less common symptoms include headache (15.4\%), pharyngalgia (13.1\%), diarrhoea (12.9\%), shivering (10.9\%), nausea and vomiting (10.2\%), and abdominal pain (4.4\%). ${ }^{1}$

While most of the cases present with mild to moderate disease, some progress to severe pneumonia and acute hypoxemic respiratory failure. Interestingly, with the progression of the pandemic, extrapulmonary manifestations involving brain and cardiac tissues were increasingly recognized. The reverse transcriptase-polymerase chain reaction (rRT-PCR) test remains the gold standard method for diagnosis. Respiratory specimens, most frequently nasopharyngeal swabs or gene sequencing are used for the same. This test is very specific, but the sensitivity is lower depending on specimen collection or viral burden at the time of sampling and thus the initial tests may be negative. ${ }^{2}$

Chest computed tomography (CT) scans play a major role in diagnosing Covid-19 pneumonia. Its diagnostic value lies in the detection and judgment of the nature of lesions, assessing the severity of the disease and further facilitating the clinical classification. A previous study showed that a patient with epidemiological history with typical Covid-19 pneumonia lesions on a CT chest scan was rendered negative multiple times by the rRT-PCR test before the final diagnosis was made. Thus, recognizing the role of CT in the early stages of the infection is vital in one's clinical course of the disease.

The typical manifestations of Covid-19 pneumonia during the early stage of a CT scan include multiple small patchy shadows and interstitial inflammation, predominantly distributed in the peripheral third of the lungs. Eventually, it develops into multiple ground-glass opacities and infiltrates the lungs. Furthermore, pulmonary consolidation was observed, but pleural effusion was rare. From initial diagnosis to patient recovery, CT scans showed significant morphological changes in the lesions, but no literature has reported small cavities in the lungs on chest CTs as a sign of Covid-19. We present the case of a 50-year-old patient with Covid-19 pneumonia who had typical manifestations of the disease on a CT scan along with constantly evolving small cavities in the lungs. ${ }^{3}$

Similar to those in chest radiography, the most suspicious CT features for Covid-19 infection in the lungs include multifocal bilateral peripheral GGOs, with or without focal consolidations, in lung regions close to pleural surfaces, including the fissures.

Ground glass opacities are defined as ill-defined hazy areas showing increased attenuation in which the bronchial, as well as vascular margins, appear intact. Consolidation is defined as intra alveolar air being replaced by abnormal fluid and tissues due to an increase in attenuation, making bronchial and vascular margins obscured. Crazy paving is thickened interlobular septa with superimposition on surrounding ground-glass opacity. Air bronchograms referred to an air attenuation bronchus on a background of the hyperattenuating opaque atelectatic lung. Subpleural lines are thin curvilinear opacities having a maximum width of 1 to $3 \mathrm{~mm}$ and located within a distance of $1 \mathrm{~cm}$ from the pleural surface and parallel to the adjacent pleural surface. 4 
Although subpleural involvement has been frequently documented, subpleural sparing can also be present. There are two types of GGOs that have been described: a pure type and a mixed pattern, which is characterized by the presence of both GGOs and areas of consolidation. On CT, GGOs with or without consolidation are typically depicted in a peripheral, posterior, and diffuse or lower-lung zone distribution. The most commonly observed patterns are pure-type GGOs, usually developing between days 0 and 4 after symptom onset, peaking at $6-13$ days. Therefore, a negative chest CT examination should not be used to exclude the possibility of Covid-19, particularly in the early phase of the disease process. $^{5}$

As radiologists, it is important to understand the natural temporal evolution of lung abnormalities in Covid-19 in determining the stage of disease and distinguishing them from potential complications of covid pneumonia. It should also be noted that there are relatively few studies that have evaluated serial temporal changes in patients with repeat CT examinations and additionally, these studies are limited by selection bias and potential confounding of the natural course of lung abnormalities owing to medical interventions (such as the administration of antimicrobial agents, fluid, or steroid therapy).

Four stages of Covid-19 in chest CT have been described: (a) early stage ( 0 - 5 days after symptom onset), which is characterized by either normal findings or mainly ground-glass opacities; (b) progressive stage (5 - 8 days after symptom onset), which is characterized by increased groundglass opacities and crazy-paving appearance; (c) peak stage ( 9 - 13 days after symptom onset), which is characterized by progressive consolidation; and (d) late stage ( $\geq 14$ days after symptom onset), which is characterized by a gradual decrease of consolidation and ground-glass opacities, while signs of fibrosis (including parenchymal bands, architectural distortion, and traction bronchiectasis) may manifest. It has been reported that unilateral involvement is only present in the early and late phases of the disease. It is also interesting to see that the temporal evolution and extent of lung abnormalities are heterogeneous among different patients, depending on the severity of the disease. ${ }^{1}$

Previous reports and our experience have revealed that Covid-19 involves multiple lobes of the lung, with typical CT findings including ground-glass opacities (GGO), opacities with rounded morphology, consolidation with GGO, and crazy-paving pattern. In addition, enlarged mediastinal lymph nodes and pleural fluid have been reported as rare CT features in some severe patients. However, as far as we know, no necrotic cavity has been reported so far. Therefore, Covid19 could be excluded if cavitary lesions are present, and this had been our mantra in the diagnosis of covid-19 until a case appeared. 6

Cavitary lung disease caused by Covid-19 viral pneumonia is not a well-recognized entity and causes of cavitary lung disease include infectious, neoplastic, vasculitis and congenital involvement of the lung. Our rigorous workup excluded many aetiologies for cavitary disease. There was no evidence for neoplasm and rapid changes were not consistent with congenital aetiologies. ${ }^{7}$

Cavitary lung lesions are usually related to mycobacterial, parasitic, fungal, autoimmune, or neoplastic aetiologies. Typical CT imaging features of Covid-19 mainly include ground-glass and consolidative pulmonary opacities, primarily in the lower lobes. Notably, there is also the absence of cavitation, lymphadenopathy, and pleural effusion. Lung cavitation following pulmonary embolism and infarction has been described previously in non-covid-19 patients. In our case, cavitation occurred in bilateral lung fields, more in the right lung. The velocity of the development of multiple cavitary lesions in a few weeks was found to be atypical for Mycobacterium tuberculosis or fungal infections such as aspergillosis and directed towards possible complications from Covid-19 pneumonia.

Lung cavitation due to Covid-19 pneumonia is uncommon. Although the exact mechanism of cavitation in Covid-19 pneumonia is unknown, it may be related to diffuse alveolar damage, intra-alveolar haemorrhage and necrosis of parenchymal cells based on prior autopsy reports. While most cases are self-limited and managed conservatively, as in our case, respiratory status must be monitored closely in patients with massive haemoptysis.

The clinical spectrum of disease secondary to SARS-CoV-2 continues to evolve. Early and late complications associated with Covid-19 are still unknown. Common causes of cavitary lung lesions must be investigated appropriately in all patients. Clinicians must be aware of evolving CT findings of Covid-19 and must arrange appropriate follow-up of convalescent patients with Covid-19 to ensure complete recovery. ${ }^{8}$

\section{CONCLUSIONS}

Cavitations are usually not seen in the case of viral pneumonia routinely; henceforth clinicians should be kept updated and made aware of radiological findings of Covid-19 pneumonia since they are continuously evolving.

This case report highlights that pulmonary cavitation in patients with severe Covid-19 lung disease can occur and leads to secondary complications and may confer a poor prognosis. Cross-sectional imaging in early stages should be considered if there is suspicion of cavitation on plain radiographs, and a more aggressive approach for investigation and treatment should be made.

Financial or other competing interests: None.

Disclosure forms provided by the authors are available with the full text of this article at jemds.com.

\section{REFERENCES}

[1] Kwee TC, Kwee RM. Chest CT in COVID-19: what the radiologist needs to know. RadioGraphics 2020;40(7):1848-65.

[2] Erok B, Kibici K, Atca AO. Pulmonary cavitation in COVID-19 pneumonia: is it primary or secondary? African Journal of Respiratory Medicine 2020;15(1):1617.

[3] Chen J, Peng S, Zhang B, et al. An uncommon manifestation of COVID-19 pneumonia on CT scan with small cavities in the lungs: a case report. Medicine 2020;99(28):e21240. 
[4] Gaurav M, Abhilasha D, Khatib MN, et al. Spectrum of initial computed tomography findings in RT-PCR Positive patients with novel Coronavirus 2019 disease-a systematic review of 2327 cases. The Open Public Health Journal 2021;14(Suppl 1):118-27.

[5] Revzin MV, Raza S, Warshawsky R, et al. Multisystem imaging manifestations of covid-19, part 1: Viral pathogenesis and pulmonary and vascular system complications. RadioGraphics 2020;40(6):1574-99.
[6] Xu Z, Pan A, Zhou H. Rare CT feature in a COVID-19 patient: cavitation. Diagnostic and Interventional Radiology 2020;26(4):380-1.

[7] Afrazi A, Garcia-Rodriguez S, Maloney JD, et al. Cavitary lung lesions and pneumothorax in a healthy patient with active coronavirus-19 (COVID-19) viral pneumonia. Interactive Cardio Vascular and Thoracic Surgery 2021;32(1):150-2.

[8] Selvaraj V, Dapaah-Afriyie K. Lung cavitation due to COVID-19 pneumonia. BMJ Case Reports 2020;13(7):e237245. 